\title{
Smoluchowski equation with variable coefficients in perforated domains: homogenization and applications to mathematical models in medicine
}

\author{
Bruno Franchi ${ }^{1}$ and Silvia Lorenzani ${ }^{2}$ \\ 1 Dipartimento di Matematica, Università di Bologna, Piazza di Porta S. \\ Donato 5, 40126 Bologna, Italy \\ ${ }^{2}$ Dipartimento di Matematica, Politecnico di Milano, Piazza Leonardo da \\ Vinci 32, 20133 Milano, Italy
}

\section{Introduction}

This paper is devoted to the homogenization of a set of Smoluchowski's discrete diffusion-coagulation equations [16] over periodically perforated domains. This type of equations, describing the evolving densities of diffusing particles that are prone to coagulate in pairs, models various physical phenomena: the evolution of a system of solid or liquid particles suspended in a gas, polymerization, aggregation of colloidal particles, formation of stars and planets as well as biological populations, behavior of fuel mixtures in engines, etc. (see, e.g. [8], [11]). Quite often, starting from a microscopic description of a problem, we seek a macroscopic, or averaged, description. As a matter of fact, while being closer to the actual physical nature, a mathematical model for a physical system that resolves smaller scales is usually more complicated and sometimes even virtually impossible to solve. Moreover, experimental data are often available for macroscale quantities only, but not for the microscale. Therefore, for quite a long time, the key issue has been how to formulate laws on a scale that is larger than the microscale and to justify these laws on the basis of a microscopic approach. To do that, in the seventies, mathematicians have developed a new method called homogenization [6]. This method allows to perform certain limits of the solutions of partial differential equations describing media with microstructures and to determine equations which the limits are solution of. Roughly speaking, what one does is to consider media with microstructures, to average out the physical and 
chemical processes arising at the microscale and to calculate effective properties of the media on the macroscale. This is precisely what has been done in the present work, where the homogenization method has been applied to the model presented below.

Let $\Omega$ be a bounded open set in $\mathbb{R}^{N}$ with a smooth boundary $\partial \Omega$. Let $Y$ be the unit periodicity cell $\left[0,1\left[^{N}\right.\right.$ having the paving property. We perforate $\Omega$ by removing from it a set $T_{\epsilon}$ of periodically distributed holes defined as follows. Let us denote by $T$ an open subset of $Y$ with a smooth boundary $\Gamma$, such that $\bar{T} \subset \operatorname{Int} Y$. Set $Y^{*}=Y \backslash T$ which is called in the literature the solid or material part. We define $\tau(\epsilon \bar{T})$ to be the set of all translated images of $\epsilon \bar{T}$ of the form $\epsilon(k+\bar{T}), k \in \mathbb{Z}^{N}$. Then,

$$
T_{\epsilon}:=\Omega \cap \tau(\epsilon \bar{T}) .
$$

Introduce now the periodically perforated domain $\Omega_{\epsilon}$ defined by

$$
\Omega_{\epsilon}=\Omega \backslash \bar{T}_{\epsilon} .
$$

For the sake of simplicity, we make the following standard assumption on the holes [7]:

there exists a 'security' zone around $\partial \Omega$ without holes, i.e.

$$
\exists \delta>0 \text { such that } \operatorname{dist}\left(\partial \Omega, T_{\epsilon}\right) \geq \delta .
$$

Therefore, $\Omega_{\epsilon}$ is a connected set ([7]). The boundary $\partial \Omega_{\epsilon}$ of $\Omega_{\epsilon}$ is then composed of two parts. The first one is the union of the boundaries of the holes strictly contained in $\Omega$. It is denoted by $\Gamma_{\epsilon}$ and is defined by

$$
\Gamma_{\epsilon}:=\cup\{\partial(\epsilon(k+\bar{T})) \mid \epsilon(k+\bar{T}) \subset \Omega\} .
$$

The second part of $\partial \Omega_{\epsilon}$ is its fixed exterior boundary denoted by $\partial \Omega$. It is easily seen that (see [3], Eq. (3))

$$
\lim _{\epsilon \rightarrow 0} \epsilon\left|\Gamma_{\epsilon}\right|_{N-1}=|\Gamma|_{N-1} \frac{|\Omega|_{N}}{|Y|_{N}}
$$

where $|\cdot|_{N-1}$ and $|\cdot|_{N}$ are the $(N-1)$-dimensional and the $N$-dimensional Hausdorff measure, respectively.

Throughout this paper, $\epsilon$ will denote the general term of a sequence of positive reals which converges to zero. From now on, let $M \in \mathbb{N}$ be fixed. We consider in the following a system of anisotropic diffusion-coagulation equations in $\Omega_{\epsilon}$ (the socalled Smoluchowski system with diffusion) which describes the dynamics of cluster 
growth. In particular, we introduce the vector-valued function $u^{\epsilon}:[0, T] \times \Omega_{\epsilon} \rightarrow$ $\mathbb{R}^{M}, u^{\epsilon}=\left(u_{1}^{\epsilon}, \ldots, u_{M}^{\epsilon}\right)$ where the variable $u_{m}^{\epsilon} \geq 0(1 \leq m<M)$ represents the concentration of $m$-clusters, that is, clusters consisting of $m$ identical elementary particles (monomers), while $u_{M}^{\epsilon} \geq 0$ takes into account aggregations of more than $M-1$ monomers. We assume that the only reaction allowing clusters to coalesce to form larger clusters is a binary coagulation mechanism, while the movement of clusters leading to aggregation results only from a diffusion process described by a matrix $D_{m}\left(t, x, \frac{x}{\epsilon}\right)(1 \leq m \leq M)$ with non-constant coefficients. Similar results for constant diffusion matrices have been obtained in [9] (see also the comments in Section 4).

Under these assumptions, our system reads:

$$
\begin{cases}\frac{\partial u_{1}^{\epsilon}}{\partial t}-\operatorname{div}\left(D_{1}\left(t, x, \frac{x}{\epsilon}\right) \nabla_{x} u_{1}^{\epsilon}\right)+u_{1}^{\epsilon} \sum_{j=1}^{M} a_{1, j} u_{j}^{\epsilon}=0 & \text { in }[0, T] \times \Omega_{\epsilon} \\ {\left[D_{1}\left(t, x, \frac{x}{\epsilon}\right) \nabla_{x} u_{1}^{\epsilon}\right] \cdot n=0} & \text { on }[0, T] \times \partial \Omega \\ {\left[D_{1}\left(t, x, \frac{x}{\epsilon}\right) \nabla_{x} u_{1}^{\epsilon}\right] \cdot n=\epsilon \psi\left(t, x, \frac{x}{\epsilon}\right)} & \text { on }[0, T] \times \Gamma_{\epsilon} \\ u_{1}^{\epsilon}(0, x) \equiv U_{1}>0 & \text { in } \Omega_{\epsilon} ;\end{cases}
$$

if $1<m<M$,

$$
\begin{cases}\frac{\partial u_{m}^{\epsilon}}{\partial t}-\operatorname{div}\left(D_{m}\left(t, x, \frac{x}{\epsilon}\right) \nabla_{x} u_{m}^{\epsilon}\right)+u_{m}^{\epsilon} \sum_{j=1}^{M} a_{m, j} u_{j}^{\epsilon}=f_{m}^{\epsilon} & \text { in }[0, T] \times \Omega_{\epsilon} \\ {\left[D_{m}\left(t, x, \frac{x}{\epsilon}\right) \nabla_{x} u_{m}^{\epsilon}\right] \cdot n=0} & \text { on }[0, T] \times \partial \Omega \\ {\left[D_{m}\left(t, x, \frac{x}{\epsilon}\right) \nabla_{x} u_{m}^{\epsilon}\right] \cdot n=0} & \text { on }[0, T] \times \Gamma_{\epsilon} \\ u_{m}^{\epsilon}(0, x)=0 & \text { in } \Omega_{\epsilon}\end{cases}
$$

and eventually

$$
\begin{cases}\frac{\partial u_{M}^{\epsilon}}{\partial t}-\operatorname{div}\left(D_{M}\left(t, x, \frac{x}{\epsilon}\right) \nabla_{x} u_{M}^{\epsilon}\right)=g^{\epsilon} & \text { in }[0, T] \times \Omega_{\epsilon} \\ {\left[D_{M}\left(t, x, \frac{x}{\epsilon}\right) \nabla_{x} u_{M}^{\epsilon}\right] \cdot n=0} & \text { on }[0, T] \times \partial \Omega \\ {\left[D_{M}\left(t, x, \frac{x}{\epsilon}\right) \nabla_{x} u_{M}^{\epsilon}\right] \cdot n=0} & \text { on }[0, T] \times \Gamma_{\epsilon} \\ u_{M}^{\epsilon}(0, x)=0 & \text { in } \Omega_{\epsilon},\end{cases}
$$

where the gain terms $f_{m}^{\epsilon}$ and $g^{\epsilon}$ in (4) and (5) are given by

$$
f_{m}^{\epsilon}=\frac{1}{2} \sum_{j=1}^{m-1} a_{j, m-j} u_{j}^{\epsilon} u_{m-j}^{\epsilon} \quad \text { and } \quad g^{\epsilon}=\frac{1}{2} \sum_{\substack{j+k \geq M \\ k<M \\ j<M}} a_{j, k} u_{j}^{\epsilon} u_{k}^{\epsilon}
$$


The kinetic coefficients $a_{i, j}$ represent a reaction in which an $(i+j)$-cluster is formed from an $i$-cluster and a $j$-cluster. Therefore, they can be interpreted as "coagulation rates" and are symmetric $a_{i, j}=a_{j, i}>0, i, j=1, \ldots, M$, but $a_{M, M}=0$. Let us remark that the meaning of $u_{M}^{\epsilon}$ differs from that of $u_{m}^{\epsilon}(m<M)$, since it describes the sum of the densities of all the 'large' assemblies. It is assumed that large assemblies exhibit all the same coagulation properties and do not coagulate with each other.

Here

$$
(t, x, y) \in[0, T] \times \Omega \times Y \rightarrow D_{m}(t, x, y)
$$

is a matrix-valued map with entries $d_{i, j}^{m}$ that are continuously differentiable in $[0, T] \times$ $\bar{\Omega} \times Y$ for $i, j=1, \ldots, N, m=1, \ldots, M$, and are $y$-periodic on $Y$. We put $\Lambda^{\star}:=$ $\max _{i, j . m}\left\|d_{i, j}^{m}\right\|_{C^{1}([0, T] \times \bar{\Omega} \times Y)}$. In addition we assume

(H.1) $(t, x) \rightarrow D_{m}\left(t, x, \frac{x}{\epsilon}\right) \quad$ is measurable on $\Omega_{\epsilon}$, and

$$
\lim _{\epsilon \rightarrow 0} \int_{0}^{T} \int_{\Omega_{\epsilon}}\left|d_{i, j}^{m}\left(t, x, \frac{x}{\epsilon}\right)\right|^{2} d t d x=\int_{0}^{T} \int_{\Omega_{\epsilon}} \int_{Y}\left|d_{i, j}^{m}(t, x, y)\right|^{2} d t d x d y
$$

(H.2) $d_{i, j}^{m}=d_{j, i}^{m}$, for $i, j=1, \ldots, N, m=1, \ldots, M$;

(H.3) there exists $0<\lambda \leq \Lambda$ such that

$$
\lambda|\xi|^{2} \leq \sum_{i, j=1}^{N} d_{i, j}^{m} \xi_{i} \xi_{j} \leq \Lambda|\xi|^{2}
$$

for all $\xi \in \mathbb{R}^{N}, m=1, \ldots, M$.

Moreover, $\psi$, appearing in (3), is a given bounded function satisfying the following conditions:

(H.4) $\psi\left(t, x, \frac{x}{\epsilon}\right) \in C^{1}(0, T ; B)$ with $B=C^{1}\left[\bar{\Omega} ; C_{\#}^{1}(Y)\right]$, where $C_{\#}^{1}(Y)$ is the subset of $C^{1}\left(\mathbb{R}^{N}\right)$ of $Y$-periodic functions;

(H.5) $\psi\left(t=0, x, \frac{x}{\epsilon}\right)=0$

and $U_{1}$ is a positive constant such that

$$
U_{1} \leq\|\psi\|_{L^{\infty}(0, T ; B)}
$$

In the Section 2 we show preliminarily that the system (3) - (5) has a unique classic solution $u^{\epsilon} \in C^{1+\alpha / 2,2+\alpha}\left([0, T] \times \Omega_{\epsilon}, \mathbb{R}^{M}\right)$ for any $\epsilon>0$. The core of this note is the study of the asymptotic behavior of $u^{\epsilon}$ as $\epsilon \rightarrow 0$ in the framework of the so-called two-scale convergence. This method, introduced by Gabriel Nguetseng [14] and Gregoire Allaire [2], relies on the following compactness theorem: 
Theorem 1.1. Let $\left(v^{\epsilon}\right)_{\epsilon>0}$ be a bounded sequence in $L^{2}([0, T] \times \Omega)$. There exists a subsequence, still denoted by $\left(v^{\epsilon}\right)_{\epsilon>0}$, and a function $v_{0}(t, x, y)$ in $L^{2}([0, T] \times \Omega \times Y)$ such that

$$
\lim _{\epsilon \rightarrow 0} \int_{0}^{T} \int_{\Omega} v^{\epsilon}(t, x) \phi\left(t, x, \frac{x}{\epsilon}\right) d t d x=\int_{0}^{T} \int_{\Omega} \int_{Y} v_{0}(t, x, y) \phi(t, x, y) d t d x d y
$$

for all $\phi \in C^{1}\left([0, T] \times \bar{\Omega} ; C_{\#}^{\infty}(Y)\right)$.

A sequence $\left(v^{\epsilon}\right)_{\epsilon>0}$ satisfying (9) is said to two-scale converge to $v_{0}(t, x, y)$.

Within the general setting of two-scale convergence, we can state our main homogenization result:

Theorem 1.2. Let $u_{m}^{\epsilon}(t, x)(1 \leq m \leq M)$ be a family of classical solutions to problems (3)-(5). Denote by a tilde the extension by zero outside $\Omega_{\epsilon}$ of a function defined in $\Omega_{\epsilon}$ and let $\chi(y)$ represent the characteristic function of $Y^{*}$.

Then, the sequences $\left(\widetilde{u_{m}^{\epsilon}}\right)_{\epsilon>0}$ and $\left(\widetilde{\nabla_{x} u_{m}^{\epsilon}}\right)_{\epsilon>0}(1 \leq m \leq M)$ two-scale converge to: $\left[\chi(y) u_{m}(t, x)\right]$ and $\left[\chi(y)\left(\nabla_{x} u_{m}(t, x)+\nabla_{y} u_{m}^{1}(t, x, y)\right)\right](1 \leq m \leq M)$, respectively, The limiting functions $\left(u_{m}(t, x), u_{m}^{1}(t, x, y)\right)(1 \leq m \leq M)$ are the unique solutions in $L^{2}\left(0, T ; H^{1}(\Omega)\right) \times L^{2}\left([0, T] \times \Omega ; H_{\#}^{1}(Y) / \mathbb{R}\right)$ of the following two-scale homogenized systems:

If $m=1$ we have:

$$
\begin{aligned}
& \begin{cases}\theta \frac{\partial u_{1}}{\partial t}(t, x)-d i v_{x}\left[D_{1}^{\star}(t, x) \nabla_{x} u_{1}(t, x)\right]+\theta u_{1}(t, x) \sum_{j=1}^{M} a_{1, j} u_{j}(t, x) & \\
=\int_{\Gamma} \psi(t, x, y) d \sigma(y) & \text { in }[0, T] \times \Omega \\
{\left[D_{1}^{\star}(t, x) \nabla_{x} u_{1}(t, x)\right] \cdot n=0} & \text { on }[0, T] \times \partial \Omega \\
u_{1}(0, x)=U_{1} & \text { in } \Omega\end{cases} \\
& \begin{array}{ll}
\text { if } 1<m<M \text { we have } & \\
\theta \frac{\partial u_{m}}{\partial t}(t, x)-d i v_{x}\left[D_{m}^{\star}(t, x) \nabla_{x} u_{m}(t, x)\right]+\theta u_{m}(t, x) \sum_{j=1}^{M} a_{m, j} u_{j}(t, x) & \text { in }[0, T] \times \Omega \\
=\frac{\theta}{2} \sum_{j=1}^{m-1} a_{j, m-j} u_{j}(t, x) u_{m-j}(t, x) &
\end{array} \\
& \begin{cases}{\left[D_{m}^{\star}(t, x) \nabla_{x} u_{m}(t, x)\right] \cdot n=0} & \text { on }[0, T] \times \partial \Omega \\
u_{m}(0, x)=0 & \text { in } \Omega\end{cases}
\end{aligned}
$$


if $m=M$ we have:

$$
\begin{cases}\theta \frac{\partial u_{M}}{\partial t}(t, x)-\operatorname{div}_{x}\left[D_{M}^{\star}(t, x) \nabla_{x} u_{M}(t, x)\right] & \\ =\frac{\theta}{2} \sum_{\substack{j+k \geq M \\ k<M \\ j<M}} a_{j, k} u_{j}(t, x) u_{k}(t, x) & \text { in }[0, T] \times \Omega \\ {\left[D_{M}^{\star}(t, x) \nabla_{x} u_{M}(t, x)\right] \cdot n=0} & \text { on }[0, T] \times \partial \Omega \\ u_{M}(0, x)=0 & \text { in } \Omega\end{cases}
$$

where

$$
\begin{gathered}
u_{m}^{1}(t, x, y)=\sum_{i=1}^{N} w_{i}(t, x, y) \frac{\partial u_{m}}{\partial x_{i}}(t, x) \quad(1 \leq m \leq M), \\
\theta=\int_{Y} \chi(y) d y=\left|Y^{*}\right|
\end{gathered}
$$

is the volume fraction of material, and $D_{m}^{\star}(t, x)$ is a matrix defined by

$$
\left(D_{m}^{\star}\right)_{i j}(t, x)=\int_{Y^{*}} D_{m}(t, x, y)\left(\nabla_{y} w_{i}(t, x, y)+\hat{e}_{i}\right) \cdot\left(\nabla_{y} w_{j}(t, x, y)+\hat{e}_{j}\right) d y
$$

with $\hat{e}_{i}$ being the $i$-th unit vector in $\mathbb{R}^{N}$, and $\left(w_{i}\right)_{1 \leq i \leq N}$ the family of solutions of the cell problem

$$
\left\{\begin{array}{lc}
-\operatorname{div}_{y}\left(D_{m}(t, x, y)\left[\nabla_{y} w_{i}(t, x, y)+\hat{e}_{i}\right]\right)=0 & \text { in } Y^{*} \\
D_{m}(t, x, y)\left[\nabla_{y} w_{i}(t, x, y)+\hat{e}_{i}\right] \cdot n=0 & \text { on } \Gamma \\
y \rightarrow w_{i}(t, x, y) \quad Y-\text { periodic } &
\end{array}\right.
$$

\section{The problem at $\epsilon$-scale: existence and regularity}

The system (3)-(5) admits a local positive classical solution. Indeed, by [4] and the usual parabolic comparison principle, we have:

Theorem 2.1. Suppose (H.1) - (H.5) hold. If $\epsilon>0$, then the system (3)-(5) admits a unique maximal classical solution $u^{\epsilon}=\left(u_{1}^{\epsilon}, \ldots, u_{M}^{\epsilon}\right)$, that is defined in a relatively open interval $J \subset[0, T]$ such that $0 \in J$. More precisely,

$$
u^{\epsilon} \in C^{0}\left(J \times \bar{\Omega}_{\epsilon}\right) \cap C^{1}\left((J \backslash\{0\}) \times \bar{\Omega}_{\epsilon}\right) \cap C^{2}\left((J \backslash\{0\}) \times \Omega_{\epsilon}\right) .
$$


Moreover

$$
u_{j}^{\epsilon}(t, x)>0 \quad \text { for }(t, x) \in(J \backslash\{0\}) \times \Omega_{\epsilon}, j=1, \ldots, M .
$$

We are now faced with several questions that will turn out to be deeply interconnected. In particular, we want to show that

- for fixed $\epsilon>0$, the local solution $u^{\epsilon}$ is in fact a global solution on $[0, T]$;

- $u^{\epsilon}$ satisfies sharp regularity estimates, i.e. $u_{j}^{\epsilon} \in C^{1+\alpha / 2,2+\alpha}([0, T] \times \Omega)$ for $j=1, \ldots, M$.

Moreover, in order to establish our homogenization results, we have to prove

- a priori estimates for the sequences $\left(u_{j}^{\epsilon}\right)_{\epsilon>0},\left(\nabla_{x} u_{j}^{\epsilon}\right)_{\epsilon>0},\left(\partial_{t} u_{j}^{\epsilon}\right)_{\epsilon>0}$ in $L^{2}([0, T] \times$ $\Omega_{\epsilon}$ ), that are independent of $\epsilon$.

The first and crucial step will consist of proving that the $u_{j}^{\epsilon}$ are equibounded in $L^{\infty}\left([0, T] \times \Omega_{\epsilon}\right)$ for $j=1, \ldots, M$. The uniform boundedness of $u_{1}^{\epsilon}(t, x)$ in $L^{\infty}([0, T] \times$ $\left.\Omega_{\epsilon}\right)$ is provided by the following statement:

Theorem 2.2. Take $0<T_{\max }<\sup J$ and let $u_{1}^{\epsilon}$ be a classical solution of (3). Then,

$$
\left\|u_{1}^{\epsilon}\right\|_{L^{\infty}\left(0, T_{\max } ; L^{\infty}\left(\Omega_{\epsilon}\right)\right)} \leq\left|U_{1}\right|+c\|\psi\|_{L^{\infty}\left(0, T_{\max } ; B\right)},
$$

where $c$ is independent of $\epsilon$.

Proof. Since

$$
\operatorname{div}\left(D_{1}\left(t, x, \frac{x}{\epsilon}\right) \nabla_{x} u_{1}^{\epsilon}\right)-\frac{\partial u_{1}^{\epsilon}}{\partial t} \geq 0,
$$

by the classical maximum principle the following estimate holds:

$$
\left\|u_{1}^{\epsilon}\right\|_{L^{\infty}\left(0, T_{\max } ; L^{\infty}\left(\Omega_{\epsilon}\right)\right)} \leq\left|U_{1}\right|+\left\|u_{1}^{\epsilon}\right\|_{L^{\infty}\left(0, T_{\max } ; L^{\infty}\left(\Gamma_{\epsilon}\right)\right)} .
$$

Thus, (14) will follow once we prove that

$$
\left\|u_{1}^{\epsilon}\right\|_{L^{\infty}\left(0, T_{\max } ; L^{\infty}\left(\Gamma_{\epsilon}\right)\right)} \leq c\|\psi\|_{L^{\infty}\left(0, T_{\max } ; B\right)}
$$

Let now $k \geq 0$ be fixed. Define: $u_{\epsilon}^{(k)}(t):=\left(u_{1}^{\epsilon}(t)-k\right)_{+}$for $t \geq 0$, with derivatives:

$$
\begin{gathered}
\frac{\partial u_{\epsilon}^{(k)}}{\partial t}=\frac{\partial u_{1}^{\epsilon}}{\partial t} \mathbb{1}_{\left\{u_{1}^{\epsilon}>k\right\}} \\
\nabla_{x} u_{\epsilon}^{(k)}=\nabla_{x} u_{1}^{\epsilon} \mathbb{1}_{\left\{u_{1}^{\epsilon}>k\right\}} .
\end{gathered}
$$


Moreover,

$$
\begin{gathered}
\left.u_{\epsilon}^{(k)}\right|_{\partial \Omega}=\left(\left.u_{1}^{\epsilon}\right|_{\partial \Omega}-k\right)_{+} \\
\left.u_{\epsilon}^{(k)}\right|_{\Gamma_{\epsilon}}=\left(\left.u_{1}^{\epsilon}\right|_{\Gamma_{\epsilon}}-k\right)_{+}
\end{gathered}
$$

Let us assume $k \geq \hat{k}$, where $\hat{k}:=\|\psi\|_{L^{\infty}\left(0, T_{\max } ; B\right)}$. Then, by (8),

$$
u_{1}^{\epsilon}(0, x)=U_{1} \leq \hat{k} \leq k
$$

For $t \in\left[0, T_{1}\right]$ with $T_{1} \leq T_{\max }$, we get

$$
\begin{aligned}
\frac{1}{2} \int_{\Omega_{\epsilon}}\left|u_{\epsilon}^{(k)}(t)\right|^{2} d x & =\int_{0}^{t} \frac{d}{d s}\left[\frac{1}{2} \int_{\Omega_{\epsilon}}\left|u_{\epsilon}^{(k)}(s)\right|^{2} d x\right] d s \\
& =\int_{0}^{t} d s \int_{\Omega_{\epsilon}} \frac{\partial u_{\epsilon}^{(k)}(s)}{\partial s} u_{\epsilon}^{(k)}(s) d x .
\end{aligned}
$$

Taking into account (3), (17) and (18), we obtain that for all $s \in\left[0, T_{1}\right]$

$$
\begin{aligned}
& \int_{\Omega_{\epsilon}} \frac{\partial u_{\epsilon}^{(k)}(s)}{\partial s} u_{\epsilon}^{(k)}(s) d x=\int_{\Omega_{\epsilon}} \frac{\partial u_{1}^{\epsilon}(s)}{\partial s} u_{\epsilon}^{(k)}(s) d x \\
& =\int_{\Omega_{\epsilon}}\left[\operatorname{div}\left(D_{1}\left(s, x, \frac{x}{\epsilon}\right) \nabla_{x} u_{1}^{\epsilon}\right)-u_{1}^{\epsilon} \sum_{j=1}^{M} a_{1, j} u_{j}^{\epsilon}\right] u_{\epsilon}^{(k)}(s) d x \\
& =-\int_{\Omega_{\epsilon}} u_{1}^{\epsilon}(s) \sum_{j=1}^{M} a_{1, j} u_{j}^{\epsilon}(s) u_{\epsilon}^{(k)}(s) d x+\epsilon \int_{\Gamma_{\epsilon}} \psi\left(s, x, \frac{x}{\epsilon}\right) u_{\epsilon}^{(k)}(s) d \sigma_{\epsilon}(x) \\
& -\int_{\Omega_{\epsilon}}\left\langle D_{1}\left(s, x, \frac{x}{\epsilon}\right) \nabla_{x} u_{\epsilon}^{(k)}(s), \nabla_{x} u_{\epsilon}^{(k)}(s)\right\rangle d x
\end{aligned}
$$

By the assumption (H.3) and Lemma 7.1 in [9], one has

$$
\begin{aligned}
& \int_{\Omega_{\epsilon}} \frac{\partial u_{\epsilon}^{(k)}(s)}{\partial s} u_{\epsilon}^{(k)}(s) d x \leq \epsilon \int_{\Gamma_{\epsilon}} \psi\left(s, x, \frac{x}{\epsilon}\right) u_{\epsilon}^{(k)}(s) d \sigma_{\epsilon}(x)-\lambda \int_{\Omega_{\epsilon}}\left|\nabla_{x} u_{\epsilon}^{(k)}(s)\right|^{2} d x \\
& \leq \frac{\epsilon}{2} \int_{B_{k}^{\epsilon}(s)}\left|\psi\left(s, x, \frac{x}{\epsilon}\right)\right|^{2} d \sigma_{\epsilon}(x)+\frac{C_{1}}{2} \int_{A_{k}^{\epsilon}(s)}\left|u_{\epsilon}^{(k)}(s)\right|^{2} d x \\
& -\left(\lambda-\frac{C_{1} \epsilon^{2}}{2}\right) \int_{\Omega_{\epsilon}}\left|\nabla_{x} u_{\epsilon}^{(k)}(s)\right|^{2} d x
\end{aligned}
$$

where we denote by $A_{k}^{\epsilon}(t)$ and $B_{k}^{\epsilon}(t)$ the set of points in $\Omega_{\epsilon}$ and on $\Gamma_{\epsilon}$, respectively, at which $u_{1}^{\epsilon}(t, x)>k$. It holds: 


$$
\begin{aligned}
& \left|A_{k}^{\epsilon}(t)\right| \leq\left|\Omega_{\epsilon}\right| \\
& \left|B_{k}^{\epsilon}(t)\right| \leq\left|\Gamma_{\epsilon}\right|
\end{aligned}
$$

with $|\cdot|$ being the Hausdorff measure.

Plugging (24) into (22) and varying over $t$, we arrive at the estimate:

$$
\begin{aligned}
& \sup _{0 \leq t \leq T_{1}}\left[\frac{1}{2} \int_{\Omega_{\epsilon}}\left|u_{\epsilon}^{(k)}(t)\right|^{2} d x\right]+\left(\lambda-\frac{C_{1} \epsilon^{2}}{2}\right) \int_{0}^{T_{1}} d t \int_{\Omega_{\epsilon}}\left|\nabla u_{\epsilon}^{(k)}(t)\right|^{2} d x \\
& \leq \frac{C_{1}}{2} \int_{0}^{T_{1}} d t \int_{A_{k}^{\epsilon}(t)}\left|u_{\epsilon}^{(k)}(t)\right|^{2} d x+\frac{\epsilon}{2} \int_{0}^{T_{1}} d t \int_{B_{k}^{\epsilon}(t)}\left|\psi\left(t, x, \frac{x}{\epsilon}\right)\right|^{2} d \sigma_{\epsilon}(x)
\end{aligned}
$$

Introducing the following norm

$$
\|u\|_{Q_{\epsilon}\left(T_{\max }\right)}^{2}:=\sup _{0 \leq t \leq T_{\max }} \int_{\Omega_{\epsilon}}|u(t)|^{2} d x+\int_{0}^{T_{\max }} d t \int_{\Omega_{\epsilon}}|\nabla u(t)|^{2} d x
$$

the inequality (25) can be rewritten as follows

$$
\begin{aligned}
\min \left\{\frac{1}{2},\left(\lambda-\frac{C_{1} \epsilon^{2}}{2}\right)\right\}\left\|u_{\epsilon}^{(k)}\right\|_{Q_{\epsilon}\left(T_{1}\right)}^{2} & \leq \frac{C_{1}}{2} \int_{0}^{T_{1}} d t \int_{A_{k}^{\epsilon}(t)}\left|u_{\epsilon}^{(k)}(t)\right|^{2} d x \\
& +\frac{\epsilon}{2} \int_{0}^{T_{1}} d t \int_{B_{k}^{\epsilon}(t)}\left|\psi\left(t, x, \frac{x}{\epsilon}\right)\right|^{2} d \sigma_{\epsilon}(x)
\end{aligned}
$$

We estimate the right-hand side of (27). From Hölder's inequality we obtain

$$
\int_{0}^{T_{1}} d t \int_{A_{k}^{\epsilon}(t)}\left|u_{\epsilon}^{(k)}(t)\right|^{2} d x \leq\left\|u_{\epsilon}^{(k)}\right\|_{L^{\bar{r}_{1}}\left(0, T_{1} ; L^{\bar{q}_{1}}\left(\Omega_{\epsilon}\right)\right)}^{2}\left\|\mathbb{1}_{A_{k}^{\epsilon}}\right\|_{L^{r_{1}^{\prime}\left(0, T_{1} ; L^{q_{1}^{\prime}}\left(\Omega_{\epsilon}\right)\right)}}
$$

with $r_{1}^{\prime}=\frac{r_{1}}{r_{1}-1}, q_{1}^{\prime}=\frac{q_{1}}{q_{1}-1}, \bar{r}_{1}=2 r_{1}, \bar{q}_{1}=2 q_{1}$, where, for $N>2, \bar{r}_{1} \in(2, \infty)$ and $\bar{q}_{1} \in\left(2, \frac{2 N}{(N-2)}\right)$ have been chosen such that

$$
\frac{1}{\bar{r}_{1}}+\frac{N}{2 \bar{q}_{1}}=\frac{N}{4}
$$

In particular, $r_{1}^{\prime}, q_{1}^{\prime}<\infty$, so that $(28)$ yields

$$
\int_{0}^{T_{1}} d t \int_{A_{k}^{\epsilon}(t)}\left|u_{\epsilon}^{(k)}(t)\right|^{2} d x \leq\left\|u_{\epsilon}^{(k)}\right\|_{L^{\pi_{1}}\left(0, T_{1} ; L^{\bar{q}_{1}}\left(\Omega_{\epsilon}\right)\right)}^{2}|\Omega|^{1 / q_{1}^{\prime}} T_{1}^{1 / r_{1}^{\prime}} .
$$

If we choose

$$
T_{1}^{1 / r_{1}^{\prime}}<\frac{\min \{1, \lambda\}}{2 C_{1}}|\Omega|^{-1 / q_{1}^{\prime}} \leq \frac{\min \left\{\frac{1}{2},\left(\lambda-\frac{C_{1} \epsilon^{2}}{2}\right)\right\}}{C_{1}}|\Omega|^{-1 / q_{1}^{\prime}},
$$


then from Eq.(117) in [9], it follows that

$$
\frac{C_{1}}{2} \int_{0}^{T_{1}} d t \int_{A_{k}^{\epsilon}(t)}\left|u_{\epsilon}^{(k)}(t)\right|^{2} d x \leq \frac{1}{2} \min \left\{\frac{1}{2},\left(\lambda-\frac{C_{1} \epsilon^{2}}{2}\right)\right\}\left\|u_{\epsilon}^{(k)}\right\|_{Q_{\epsilon}\left(T_{1}\right)}^{2} .
$$

Analogously, from Hölder's inequality we have, for $k \geq \hat{k}$

$$
\begin{aligned}
\frac{\epsilon}{2} \int_{0}^{T_{1}} d t \int_{B_{k}^{\epsilon}(t)}\left|\psi\left(t, x, \frac{x}{\epsilon}\right)\right|^{2} d \sigma_{\epsilon}(x) & \leq \frac{\epsilon k^{2}}{2}\left(\frac{\hat{k}^{2}}{k^{2}}\right)\left\|\mathbb{1}_{B_{k}^{\epsilon}}\right\|_{L^{1}\left(0, T_{1} ; L^{1}\left(\Gamma_{\epsilon}\right)\right)} \\
& \leq \frac{\epsilon k^{2}}{2} \int_{0}^{T_{1}} d t\left|B_{k}^{\epsilon}(t)\right| .
\end{aligned}
$$

Thus (27) yields

$$
\left\|u_{\epsilon}^{(k)}\right\|_{Q_{\epsilon}\left(T_{1}\right)}^{2} \leq \epsilon \gamma k^{2} \int_{0}^{T_{1}} d t\left|B_{k}^{\epsilon}(t)\right| .
$$

Now, as in [9] (Theorem 5.2), relying on arguments that go back to [10], [15], it follows from (32) that

$$
\left\|u_{1}^{\epsilon}\right\|_{L^{\infty}\left(0, T_{1} ; L^{\infty}\left(\Gamma_{\epsilon}\right)\right)} \leq 2 m \hat{k}
$$

where the positive constant $m$ is independent of $\epsilon$. Analogous arguments are valid for the cylinder $\left[T_{s}, T_{s+1}\right] \times \Omega_{\epsilon}, s=1,2, \ldots, p-1$ with

$$
\left[T_{s+1}-T_{s}\right]^{1 / r_{1}^{\prime}}<\frac{\min \{1, \lambda\}}{2 C_{1}}|\Omega|^{-1 / q_{1}^{\prime}}
$$

and $T_{p} \equiv T_{\max }$. Thus, after a finite number of steps, we get the estimate (16), completing the proof of Theorem 2.2.

Following the inductive argument presented in [17] (Lemma 2.2), we obtain eventually the global $L^{\infty}$ estimate for local classical solutions of (4)-(5).

Theorem 2.3. Let $u_{j}^{\epsilon}(t, x)(1 \leq j \leq M)$ be a classical solution of (3)-(5). Then there exists $K>0$ such that

$$
\left\|u_{j}^{\epsilon}\right\|_{L^{\infty}\left(0, T_{\max } ; L^{\infty}\left(\Omega_{\epsilon}\right)\right)} \leq K
$$

uniformly with respect to $\epsilon$.

A first consequence of the estimates (14) and (33) is that, for any fixed $\epsilon>0$, $J=[0, T)$ and $u^{\epsilon}$ satisfies sharp Hölder estimates.

Theorem 2.4. Let $\epsilon>0$ be fixed. Then 
i) $T_{\max }=$ T, i.e. $J=[0, T)$;

ii) there exists $\alpha \in(0,1)$, $\alpha$ depending only on $N, \lambda, \Lambda^{\star}$, and $\epsilon$, such that $u^{\epsilon} \in$ $C^{1+\alpha / 2,2+\alpha}\left([0, T] \times \Omega_{\epsilon}, \mathbb{R}^{M}\right)$ and

$$
\left\|u^{\epsilon}\right\|_{C^{1+\alpha / 2,2+\alpha}\left([0, T] \times \Omega_{\epsilon}, \mathbb{R}^{M}\right)} \leq C_{0}=C_{0}\left(U_{1},\|\psi\|_{L^{\infty}(0, T ; B)}, K, \epsilon, \alpha\right) .
$$

Proof. First of all, we notice that, if we prove (34) in $J$, then, in particular, if $N<p<\infty$, we have

$$
\left\|u^{\epsilon}\right\|_{L^{\infty}\left(J, W^{2, p}\left(\Omega_{\epsilon}\right)^{M}\right)}<\infty .
$$

Thus i) follows by [4], p. 154.

Let us prove ii). To avoid cumbersome notations, let us set

$$
F_{m}\left(t, x, u^{\epsilon}\right)= \begin{cases}-u_{1}^{\epsilon} \sum_{j=1}^{M} a_{1, j} u_{j}^{\epsilon} & \text { if } m=1 \\ -u_{m}^{\epsilon} \sum_{j=1}^{M} a_{m, j} u_{j}^{\epsilon}+f_{m}^{\epsilon} & \text { if } 2 \leq m<M \\ g^{\epsilon} & \text { if } m=M\end{cases}
$$

and $F:=\left(F_{1}, \ldots, F_{M}\right)$.

First of all, we can use a modified version for the parabolic Neumann-Cauchy problem of the classical Hölder estimates for the corresponding Dirichlet-Cauchy problem, as one can find, for instance, in [12] (Theorem 6.44). If $D[r]$ is an arbitrary parabolic cylinder

$$
D[r]=\left\{(x, t) ;\left|x-x_{0}\right|<r,\left|t-t_{0}\right|<r^{2}\right\} \cap(\Omega \times[0, T]),
$$

we obtain

$$
\operatorname{osc}_{D[r]} u_{m}^{\epsilon} \leq C r^{\alpha}\left[\sup _{[0, T] \times \Omega}\left|u_{m}^{\epsilon}\right|+\sup _{[0, T] \times \Omega}\left|F_{m}\left(t, x, u^{\epsilon}\right)\right|\right]
$$

for any $m=1, \ldots, M$ and $0<r<1$, where $C$ depends on $\Omega, T, \lambda$ and $\Lambda$. Thus, by Theorem 2.3,

$$
\begin{gathered}
\left\|u^{\epsilon}\right\|_{C^{\alpha / 2, \alpha}\left([0, T] \times \Omega_{\epsilon}, \mathbb{R}^{M}\right)} \leq \sup _{(t, x),(\tau, \xi) \in[0, T] \times \Omega} \frac{\left|u_{m}^{\epsilon}(t, x)-u_{m}^{\epsilon}(\tau, \xi)\right|}{|x-\xi|^{\alpha}+|t-\tau|^{\alpha / 2}}+K \\
\leq C\left[\sup _{[0, T] \times \Omega}\left|u^{\epsilon}\right|+\sup _{[0, T] \times \Omega}\left|F\left(t, x, u^{\epsilon}\right)\right|\right]+K \leq C\left(1+K^{2}\right) .
\end{gathered}
$$

We write now equations (3)-(5) in non-divergence form, and then we apply classic Hölder estimates as in [10] (Theorem 5.2) and [12] (Theorem 5.18). Eventually, keeping in mind (37), (34) follows. This achieves the proof of the theorem. 
We stress that all the constants involved in these Hölder estimates depend also on the space derivatives of the diffusion coefficients. Since in (3)-(5) the diffusion coefficients have the form $d_{i, j}^{m}(t, x, x / \epsilon)$, then our Hölder estimates turn out to depend on $\epsilon$.

\section{Homogenization}

In order to prove that the solutions $u^{\epsilon}$ of our Neumann-Cauchy problem at the scale $\epsilon$ converge to a solution of the homogenized problem described in Theorem 1.2 , we need a priori $L^{2}$-estimates of the derivatives of $u^{\epsilon}$, that are independent of $\epsilon>0$. Unfortunately, the bounds in (34) are not uniform in $\epsilon$, and therefore the compactness Theorem 1.1 does not apply

To overcome this difficulty, in the sequel we shall prove weaker estimates, that nevertheless are uniform in $\epsilon$.

Theorem 3.1. The sequence $\left(\nabla_{x} u_{m}^{\epsilon}\right)_{\epsilon>0}(1 \leq m \leq M)$ is bounded in $L^{2}\left([0, T] \times \Omega_{\epsilon}\right)$, uniformly in $\epsilon$.

Proof. Case $m=1$ : let us multiply the first equation in (3) by the function $u_{1}^{\epsilon}(t, x)$. Integrating, the divergence theorem yields

$$
\begin{aligned}
\frac{1}{2} \int_{\Omega_{\epsilon}} \frac{\partial}{\partial t}\left|u_{1}^{\epsilon}\right|^{2} d x & +\int_{\Omega_{\epsilon}}\left\langle D_{1}\left(t, x, \frac{x}{\epsilon}\right) \nabla_{x} u_{1}^{\epsilon}, \nabla_{x} u_{1}^{\epsilon}\right\rangle d x+\int_{\Omega_{\epsilon}}\left|u_{1}^{\epsilon}\right|^{2} \sum_{j=1}^{M} a_{1, j} u_{j}^{\epsilon} d x \\
& =\epsilon \int_{\Gamma_{\epsilon}} \psi\left(t, x, \frac{x}{\epsilon}\right) u_{1}^{\epsilon}(t, x) d \sigma_{\epsilon}(x)
\end{aligned}
$$

By the assumption (H.3) and taking into account that the third term on the left-hand side of (38) is nonnegative, one has

$$
\begin{aligned}
\frac{1}{2} \int_{\Omega_{\epsilon}} \frac{\partial}{\partial t}\left|u_{1}^{\epsilon}\right|^{2} d x & +\lambda \int_{\Omega_{\epsilon}}\left|\nabla_{x} u_{1}^{\epsilon}\right|^{2} d x \\
& \leq \epsilon \int_{\Gamma_{\epsilon}} \psi\left(t, x, \frac{x}{\epsilon}\right) u_{1}^{\epsilon}(t, x) d \sigma_{\epsilon}(x)
\end{aligned}
$$

Let us now estimate the term on the right-hand side of (39). It follows from Lemma 7.4 in [9] that

$$
\epsilon \int_{\Gamma_{\epsilon}}\left|\psi\left(t, x, \frac{x}{\epsilon}\right)\right|^{2} d \sigma_{\epsilon}(x) \leq C_{2}\|\psi(t)\|_{B}^{2}
$$

where $C_{2}$ is a positive constant independent of $\epsilon$ and $B=C^{1}\left[\bar{\Omega} ; C_{\#}^{1}(Y)\right]$. Hence, by Hölder's and Young's inequalities and Lemma 7.1 in [9], we deduce 


$$
\begin{aligned}
& \int_{\Omega_{\epsilon}} \frac{\partial}{\partial t}\left|u_{1}^{\epsilon}\right|^{2} d x+\left(2 \lambda-\epsilon^{2} C_{1}\right) \int_{\Omega_{\epsilon}}\left|\nabla_{x} u_{1}^{\epsilon}\right|^{2} d x \\
& \leq C_{2}\|\psi(t)\|_{B}^{2}+C_{1} \int_{\Omega_{\epsilon}}\left|u_{1}^{\epsilon}\right|^{2} d x
\end{aligned}
$$

Integrating over $[0, t]$ with $t \in[0, T]$, we get

$$
\left\|u_{1}^{\epsilon}(t)\right\|_{L^{2}\left(\Omega_{\epsilon}\right)}^{2}+\left(2 \lambda-\epsilon^{2} C_{1}\right) \int_{0}^{t} d s \int_{\Omega_{\epsilon}}\left|\nabla_{x} u_{1}^{\epsilon}\right|^{2} d x \leq C_{3}+C_{1}\left\|u_{1}^{\epsilon}\right\|_{L^{2}\left(0, T ; L^{2}\left(\Omega_{\epsilon}\right)\right)}^{2}
$$

where $C_{1}$ and $C_{3}$ are positive constants independent of $\epsilon$ since, by (8),

$$
u_{1}^{\epsilon}(0, x)=U_{1} \leq\|\psi\|_{L^{\infty}(0, T ; B)} .
$$

Taking into account that the first term on the left-hand side of (42) is nonnegative and the sequence $\left(u_{1}^{\epsilon}\right)_{\epsilon>0}$ is bounded in $L^{\infty}\left(0, T ; L^{\infty}\left(\Omega_{\epsilon}\right)\right)$, one has

$$
\left(2 \lambda-\epsilon^{2} C_{1}\right)\left\|\nabla_{x} u_{1}^{\epsilon}\right\|_{L^{2}\left(0, T ; L^{2}\left(\Omega_{\epsilon}\right)\right)}^{2} \leq C_{4}
$$

Thus the boundedness of $\nabla_{x} u_{1}^{\epsilon}(t, x)$ follows, provided that $\epsilon$ is close to zero.

The proof for the case $1<m \leq M$ is achieved by applying exactly the same arguments considered when $m=1$.

Theorem 3.2. The sequence $\left(\partial_{t} u_{m}^{\epsilon}\right)_{\epsilon>0}(1 \leq m \leq M)$ is bounded in $L^{2}\left([0, T] \times \Omega_{\epsilon}\right)$, uniformly in $\epsilon$.

Proof. Case $m=1$ : let us multiply the first equation in (3) by the function $\partial_{t} u_{1}^{\epsilon}(t, x)$. Integrating, the divergence theorem yields

$$
\begin{aligned}
& \int_{\Omega_{\epsilon}}\left|\frac{\partial u_{1}^{\epsilon}(t, x)}{\partial t}\right|^{2} d x+\frac{1}{2} \int_{\Omega_{\epsilon}} \frac{\partial}{\partial t}\left\langle D_{1}\left(t, x, \frac{x}{\epsilon}\right) \nabla_{x} u_{1}^{\epsilon}, \nabla_{x} u_{1}^{\epsilon}\right\rangle d x \\
& -\frac{1}{2} \int_{\Omega_{\epsilon}}\left\langle\partial_{t} D_{1} \nabla_{x} u_{1}^{\epsilon}, \nabla_{x} u_{1}^{\epsilon}\right\rangle d x+\int_{\Omega_{\epsilon}}\left(\sum_{j=1}^{M} a_{1, j} u_{1}^{\epsilon} u_{j}^{\epsilon}\right) \frac{\partial u_{1}^{\epsilon}}{\partial t} d x \\
& =\epsilon \int_{\Gamma_{\epsilon}} \psi\left(t, x, \frac{x}{\epsilon}\right) \frac{\partial u_{1}^{\epsilon}}{\partial t} d \sigma_{\epsilon}(x)
\end{aligned}
$$

From Hölder's and Young's inequalities, exploiting the boundedness of $u_{l}^{\epsilon}(t, x)(1 \leq$ $l \leq M)$ in $L^{\infty}\left(0, T ; L^{\infty}\left(\Omega_{\epsilon}\right)\right)$, one has

$$
\begin{aligned}
& \int_{\Omega_{\epsilon}}\left|\frac{\partial u_{1}^{\epsilon}(t, x)}{\partial t}\right|^{2} d x+\frac{\partial}{\partial t} \int_{\Omega_{\epsilon}}\left\langle D_{1}\left(t, x, \frac{x}{\epsilon}\right) \nabla_{x} u_{1}^{\epsilon}, \nabla_{x} u_{1}^{\epsilon}\right\rangle d x \\
& -\int_{\Omega_{\epsilon}}\left\langle\partial_{t} D_{1} \nabla_{x} u_{1}^{\epsilon}, \nabla_{x} u_{1}^{\epsilon}\right\rangle d x \leq C_{1}+2 \epsilon \int_{\Gamma_{\epsilon}} \psi\left(t, x, \frac{x}{\epsilon}\right) \frac{\partial u_{1}^{\epsilon}}{\partial t} d \sigma_{\epsilon}(x)
\end{aligned}
$$


where $C_{1}$ is a positive constant independent of $\epsilon$. By the assumption (H.3), we get

$$
\begin{aligned}
& \int_{\Omega_{\epsilon}}\left|\frac{\partial u_{1}^{\epsilon}(t, x)}{\partial t}\right|^{2} d x+\lambda \frac{\partial}{\partial t} \int_{\Omega_{\epsilon}}\left|\nabla_{x} u_{1}^{\epsilon}\right|^{2} d x \\
& \leq C_{1}+\Lambda^{\star} \int_{\Omega_{\epsilon}}\left|\nabla_{x} u_{1}^{\epsilon}\right|^{2} d x+2 \epsilon \int_{\Gamma_{\epsilon}} \psi\left(t, x, \frac{x}{\epsilon}\right) \frac{\partial u_{1}^{\epsilon}}{\partial t} d \sigma_{\epsilon}(x)
\end{aligned}
$$

Integrating over $[0, t]$ with $t \in[0, T]$, we obtain

$$
\begin{aligned}
& \int_{0}^{t} d s \int_{\Omega_{\epsilon}}\left|\frac{\partial u_{1}^{\epsilon}}{\partial s}\right|^{2} d x+\lambda \int_{\Omega_{\epsilon}}\left|\nabla_{x} u_{1}^{\epsilon}(t, x)\right|^{2} d x \leq C_{1} T \\
& +\Lambda^{\star} \int_{0}^{t} d s \int_{\Omega_{\epsilon}}\left|\nabla_{x} u_{1}^{\epsilon}\right|^{2} d x+2 \epsilon \int_{\Gamma_{\epsilon}} \psi\left(t, x, \frac{x}{\epsilon}\right) u_{1}^{\epsilon}(t, x) d \sigma_{\epsilon}(x) \\
& -2 \epsilon \int_{0}^{t} d s \int_{\Gamma_{\epsilon}} \frac{\partial}{\partial s} \psi\left(s, x, \frac{x}{\epsilon}\right) u_{1}^{\epsilon}(s, x) d \sigma_{\epsilon}(x)
\end{aligned}
$$

since $\psi\left(t=0, x, \frac{x}{\epsilon}\right) \equiv 0$. Now we estimate the last two terms on the right-hand side of (47).

From Hölder's and Young's inequalities, taking into account (40) and Lemma 7.1 in [9], one has

$$
2 \epsilon \int_{\Gamma_{\epsilon}} \psi\left(t, x, \frac{x}{\epsilon}\right) u_{1}^{\epsilon}(t, x) d \sigma_{\epsilon}(x) \leq C_{2}+\epsilon^{2} C_{3} \int_{\Omega_{\epsilon}}\left|\nabla_{x} u_{1}^{\epsilon}\right|^{2} d x
$$

where $C_{2}$ is a positive constant independent of $\epsilon$ since $\psi \in L^{\infty}(0, T ; B)$ and $u_{1}^{\epsilon}$ is bounded in $L^{\infty}\left(0, T ; L^{\infty}\left(\Omega_{\epsilon}\right)\right)$. Analogously, we get the following inequality

$$
\begin{aligned}
& 2 \epsilon \int_{0}^{t} d s \int_{\Gamma_{\epsilon}} \frac{\partial}{\partial s} \psi\left(s, x, \frac{x}{\epsilon}\right) u_{1}^{\epsilon}(s, x) d \sigma_{\epsilon}(x) \\
& \leq C_{4} T+C_{5} \int_{0}^{t} d s\left\|u_{1}^{\epsilon}(s)\right\|_{L^{2}\left(\Omega_{\epsilon}\right)}^{2}+\epsilon^{2} C_{5} \int_{0}^{t} d s\left\|\nabla_{x} u_{1}^{\epsilon}(s)\right\|_{L^{2}\left(\Omega_{\epsilon}\right)}^{2} \\
& \leq C_{6}
\end{aligned}
$$

where $C_{6} \geq 0$ is a constant independent of $\epsilon$, since $\left(u_{1}^{\epsilon}\right)_{\epsilon>0}$ is bounded in $L^{\infty}\left(0, T ; L^{\infty}\left(\Omega_{\epsilon}\right)\right)$, $\left(\nabla_{x} u_{1}^{\epsilon}\right)_{\epsilon>0}$ is bounded in $L^{2}\left(0, T ; L^{2}\left(\Omega_{\epsilon}\right)\right)$ and

$$
\epsilon \int_{\Gamma_{\epsilon}}\left|\partial_{t} \psi\left(t, x, \frac{x}{\epsilon}\right)\right|^{2} d \sigma_{\epsilon}(x) \leq \tilde{C}\left\|\partial_{t} \psi(t)\right\|_{B}^{2} \leq C_{4}
$$

with $\tilde{C}$ and $C_{4}$ independent of $\epsilon$. Combining the estimates (48) and (49) with (47) we obtain

$$
\int_{0}^{t} d s \int_{\Omega_{\epsilon}}\left|\frac{\partial u_{1}^{\epsilon}}{\partial s}\right|^{2} d x+\left(\lambda-\epsilon^{2} C_{3}\right) \int_{\Omega_{\epsilon}}\left|\nabla_{x} u_{1}^{\epsilon}\right|^{2} d x \leq C_{7}
$$


For a sequence $\epsilon$ of positive numbers going to zero: $\left(\lambda-\epsilon^{2} C_{3}\right) \geq 0$. Then, the second term on the left-hand side of (50) is nonnegative, and one has

$$
\left\|\partial_{t} u_{1}^{\epsilon}\right\|_{L^{2}\left(0, T ; L^{2}\left(\Omega_{\epsilon}\right)\right)}^{2} \leq C
$$

where $C \geq 0$ is a constant independent of $\epsilon$.

The proof for the case $1<m \leq M$ is achieved by applying exactly the same arguments considered when $m=1$.

Proof of Theorem 1.2. In view of Theorems 2.2, 2.3 and 3.1, the sequences $\left(\widetilde{u_{m}^{\epsilon}}\right)_{\epsilon>0}$ and $\left(\widetilde{\nabla_{x} u_{m}^{\epsilon}}\right)_{\epsilon>0}(1 \leq m \leq M)$ are bounded in $L^{2}([0, T] \times \Omega)$, and by application of Theorems 7.1 and 7.3 in [9], they two-scale converge, up to a subsequence, to: $\left[\chi(y) u_{m}(t, x)\right]$ and $\left[\chi(y)\left(\nabla_{x} u_{m}(t, x)+\nabla_{y} u_{m}^{1}(t, x, y)\right)\right](1 \leq m \leq M)$. Similarly, in view of Theorem 3.2, it is possible to prove that the sequence $\left(\frac{\widetilde{\partial u_{m}^{\epsilon}}}{\partial t}\right)_{\epsilon>0} \quad(1 \leq m \leq$ $M)$ two-scale converges to: $\left[\chi(y) \frac{\partial u_{m}}{\partial t}(t, x)\right](1 \leq m \leq M)$.

We can now find the homogenized equations satisfied by $u_{m}(t, x)$ and $u_{m}^{1}(t, x, y)$ $(1 \leq m \leq M)$.

Case $m=1$ : let us multiply the first equation of (3) by the test function

$$
\phi_{\epsilon} \equiv \phi(t, x)+\epsilon \phi_{1}\left(t, x, \frac{x}{\epsilon}\right)
$$

where $\phi \in C^{1}([0, T] \times \bar{\Omega})$ and $\phi_{1} \in C^{1}\left([0, T] \times \bar{\Omega} ; C_{\#}^{\infty}(Y)\right)$. Integrating, the divergence theorem yields

$$
\begin{aligned}
& \int_{0}^{T} \int_{\Omega_{\epsilon}} \frac{\partial u_{1}^{\epsilon}}{\partial t} \phi_{\epsilon}\left(t, x, \frac{x}{\epsilon}\right) d t d x+\int_{0}^{T} \int_{\Omega_{\epsilon}}\left\langle D_{1}\left(t, x, \frac{x}{\epsilon}\right) \nabla_{x} u_{1}^{\epsilon}, \nabla \phi_{\epsilon}\right\rangle d t d x \\
& +\int_{0}^{T} \int_{\Omega_{\epsilon}} u_{1}^{\epsilon} \sum_{j=1}^{M} a_{1, j} u_{j}^{\epsilon} \phi_{\epsilon} d t d x=\epsilon \int_{0}^{T} \int_{\Gamma_{\epsilon}} \psi\left(t, x, \frac{x}{\epsilon}\right) \phi_{\epsilon} d t d \sigma_{\epsilon}(x)
\end{aligned}
$$

Passing to the two-scale limit we get 


$$
\begin{aligned}
& \int_{0}^{T} \int_{\Omega} \int_{Y^{*}} \frac{\partial u_{1}}{\partial t}(t, x) \phi(t, x) d t d x d y \\
& +\int_{0}^{T} \int_{\Omega} \int_{Y^{*}} D_{1}(t, x, y)\left[\nabla_{x} u_{1}(t, x)+\nabla_{y} u_{1}^{1}(t, x, y)\right] \cdot\left[\nabla_{x} \phi(t, x)+\nabla_{y} \phi_{1}(t, x, y)\right] d t d x d y \\
& +\int_{0}^{T} \int_{\Omega} \int_{Y^{*}} u_{1}(t, x) \sum_{j=1}^{M} a_{1, j} u_{j}(t, x) \phi(t, x) d t d x d y \\
& =\int_{0}^{T} \int_{\Omega} \int_{\Gamma} \psi(t, x, y) \phi(t, x) d t d x d \sigma(y)
\end{aligned}
$$

where assumption (H.1) has been taken into account. The last term on the lefthand side of (53) has been obtained by using Theorem 7.2 in [9], while the term on the right-hand side has been attained by application of Theorem 7.5 in [9]. An integration by parts shows that (53) is a variational formulation associated to the following homogenized system:

$$
\begin{array}{cc}
-d i v_{y}\left[D_{1}(t, x, y)\left(\nabla_{x} u_{1}(t, x)+\nabla_{y} u_{1}^{1}(t, x, y)\right)\right]=0 & \text { in }[0, T] \times \Omega \times Y^{*} \\
{\left[D_{1}(t, x, y)\left(\nabla_{x} u_{1}(t, x)+\nabla_{y} u_{1}^{1}(t, x, y)\right)\right] \cdot n=0} & \text { on }[0, T] \times \Omega \times \Gamma \\
\theta \frac{\partial u_{1}}{\partial t}(t, x)-d i v_{x}\left[\int_{Y^{*}} D_{1}(t, x, y)\left(\nabla_{x} u_{1}(t, x)+\nabla_{y} u_{1}^{1}(t, x, y)\right) d y\right] \\
+\theta u_{1}(t, x) \sum_{j=1}^{M} a_{1, j} u_{j}(t, x)-\int_{\Gamma} \psi(t, x, y) d \sigma(y)=0 & \text { in }[0, T] \times \Omega \\
{\left[\int_{Y^{*}} D_{1}(t, x, y)\left(\nabla_{x} u_{1}(t, x)+\nabla_{y} u_{1}^{1}(t, x, y)\right) d y\right] \cdot n=0} & \text { on }[0, T] \times \partial \Omega
\end{array}
$$

where

$$
\theta=\int_{Y} \chi(y) d y=\left|Y^{*}\right|
$$

is the volume fraction of material. To conclude, by continuity, we have that

$$
u_{1}(0, x)=U_{1} \quad \text { in } \Omega \text {. }
$$

The function $u_{1}^{1}(t, x, y)$, satisfying (54)-(55), can be expressed as follows 


$$
u_{1}^{1}(t, x, y)=\sum_{i=1}^{N} w_{i}(t, x, y) \frac{\partial u_{1}}{\partial x_{i}}(t, x)
$$

where $\left(w_{i}\right)_{1 \leq i \leq N}$ is the family of solutions of the cell problem

$$
\begin{cases}-\operatorname{div}_{y}\left(D_{1}(t, x, y)\left[\nabla_{y} w_{i}(t, x, y)+\hat{e}_{i}\right]\right)=0 & \text { in } Y^{*} \\ D_{1}(t, x, y)\left[\nabla_{y} w_{i}(t, x, y)+\hat{e}_{i}\right] \cdot n=0 & \text { on } \Gamma \\ y \rightarrow w_{i}(t, x, y) \quad Y \text {-periodic } & \end{cases}
$$

By using the relation (58) in Eqs. (56) and (57), we get

$$
\begin{array}{cc}
\theta \frac{\partial u_{1}}{\partial t}(t, x)-\operatorname{div}_{x}\left[D_{1}^{\star} \nabla_{x} u_{1}(t, x)\right]+\theta u_{1}(t, x) \sum_{j=1}^{M} a_{1, j} u_{j}(t, x) \\
-\int_{\Gamma} \psi(t, x, y) d \sigma(y)=0 & \text { in }[0, T] \times \Omega \\
{\left[D_{1}^{\star} \nabla_{x} u_{1}(t, x)\right] \cdot n=0} & \text { on }[0, T] \times \partial \Omega
\end{array}
$$

where the entries of the matrix $D_{1}^{\star}$ are given by

$$
\left(D_{1}^{\star}\right)_{i j}(t, x)=\int_{Y^{*}} D_{1}(t, x, y)\left[\nabla_{y} w_{i}(t, x, y)+\hat{e}_{i}\right] \cdot\left[\nabla_{y} w_{j}(t, x, y)+\hat{e}_{j}\right] d y .
$$

The proof for the case $1<m \leq M$ is achieved by applying exactly the same arguments considered when $m=1$.

\section{A mathematical model in medicine}

Recently, the Smoluchowski equation with diffusion has been introduced for the study of a mathematical model in medicine ([13], [1], [5], [9]): the diffusion and the aggregation of the $\beta$-amyloid in the cerebral tissue affected by Alzheimer's Disease (AD). Nowadays, the so-called amyloid cascade hypothesis is largely accepted: roughly speaking, the $\mathrm{A} \beta$-peptide is produced normally by the intramembranous proteolysis of APP (amyloid precursor protein) throughout life, but a change in the metabolism (due to unknow reasons, partially genetic) may increase the total production of the monomeric isoform $\mathrm{A} \beta_{42}$, that is highly toxic for neurons. Thus, high concentrations of $\mathrm{A} \beta_{42}$ lead to neuronal death, synaptic degeneration and eventually to dementia. Successively, $\mathrm{A} \beta_{42}$ oligomers are subject to agglomeration (leading 
ultimately to the formation of long, insoluble amyloid fibrils, which accumulate in microscopic deposits known as senile plaques) and to diffusion through the microscopic tortuosities of the brain tissue.

Mathematically, this process can be modeled at a microscopic level through the system (3)-(5). More precisely, we define the periodically perforated domain $\Omega_{\epsilon}$, obtained by removing from the fixed domain $\Omega$ (the cerebral tissue) infinitely many small holes of size $\epsilon$ (the neurons), which support a non-homogeneous Neumann boundary condition describing the production of $\mathrm{A} \beta_{42}$ by the neuron membranes. Then, we prove that, when $\epsilon \rightarrow 0$, the solution of this micro-model two-scale converges to the solution of a macro-model asymptotically consistent with the original one. Indeed, the information given on the microscale by the non-homogeneous Neumann boundary condition is transferred into a source term appearing in the limiting (homogenized) equations. Furthermore, on the macroscale, the geometric structure of the perforated domain induces a correction in the diffusion matrix of the limit problem.

A similar approach to the transition from the microscopic model to the macroscopic one has been carried out starting from constant diffusion coefficients in [9]. Here, we have considered the case of diffusion matrices depending on time, on the macroscopic variable $x \in \Omega$ and, most of all, on the microscopic variable $y \in Y$. Indeed, aging (as well as the AD itself) yields an atrophy of the cerebral tissue, that induces changes in the diffusion rate of the amyloid fibrils. Analogously, this rate may vary for different regions of the brain. Finally, the dependence on the microscopic variable makes possible to include in the model the specific features of the diffusion. Indeed, the $\mathrm{A} \beta_{42}$-polymers do not diffuse freely in an uniform fluid: the cerebral tissue consists of large non-neuronal support cells (the macroglia) and the cerebrospinal fluid moves along the interstices between these cells.

\section{References}

[1] Y. Achdou, B. Franchi, N. Marcello and M. C. Tesi, A qualitative model for aggregation and diffusion of $\beta$-Amyloid in Alzheimer's disease. J. Math. Biol., 67: no. 6-7, 1369-1392, 2013.

[2] G. Allaire, Homogenization and two-scale convergence. Siam J. Math. Anal., 23(6): 1482-1518, 1992. 
[3] G. Allaire, A. Damlamian and U. Hornung, Two-scale convergence on periodic surfaces and applications. In: Proceedings of the International Conference on Mathematical Modelling of Flow through Porous Media. A. Bourgeat et al. eds., pp. 15-25, World Scientific pub., Singapore, 1996.

[4] H. Amann, Quasilinear parabolic systems under nonlinear boundary conditions. Arch. Rational Mech. Anal., 92: 153-192, 1986.

[5] M. Bertsch, B. Franchi, N. Marcello, M.C. Tesi and A. Tosin, Alzheimer's disease: A mathematical model for onset and progression. Math. Med. Biol., 2016.

[6] D. Cioranescu and P. Donato, An introduction to homogenization . Oxford University Press, Oxford, 1999.

[7] A. Damlamian and P. Donato, Which sequences of holes are admissible for periodic homogenization with Neumann boundary condition?. ESAIM: COCV, 8: 555-585, 2002.

[8] R. L. Drake, A general mathematical survey of the coagulation equation.. Topics in Current Aerosol Research (Part 2), International Reviews in Aerosol Physics and Chemistry. Pergamon Press, Oxford, 1972.

[9] B. Franchi and S. Lorenzani, From a microscopic to a macroscopic model for Alzheimer disease: Two-scale homogenization of the Smoluchowski equation in perforated domains. J. Nonlinear Sci., 26: 717-753, 2016.

[10] O. A. Ladyzenskaja, V. A. Solonnikov and N. N. Ural'ceva, Linear and quasilinear equations of parabolic type. American Mathematical Society, 1968.

[11] P. Laurençot and S. Mischler, Global existence for the discrete diffusive coagulation-fragmentation equations in $L^{1}$. Rev. Mat. Iberoamericana, 18: 731$745,2002$.

[12] G. M. Lieberman, Second order parabolic differential equations. World Scientific (1996).

[13] R. M. Murphy and M. M. Pallitto, Probing the kinetics of $\beta$-amyloid selfassociation. Journal of Structural Biology, 130: 109-122, 2000.

[14] G. Nguetseng, A general convergence result for a functional related to the theory of homogenization. Siam J. Math. Anal., 20: 608-623, 1989. 
[15] R. Nittka, Inhomogeneous parabolic Neumann problems. Czechoslovak Mathematical Journal, 64: 703-742, 2014.

[16] M. Smoluchowski, Versuch einer mathematischen theorie der koagulationskinetik kolloider lsungen. IZ Phys. Chem., 92: 129-168, 1917.

[17] D. Wrzosek, Existence of solutions for the discrete coagulation-fragmentation model with diffusion. Topol. Methods Nonlinear Anal., 9(2): 279-296, 1997. 